\title{
Incidence and risk factors of pediatric asthma exacerbations in a multinational, multidatabase cohort study
}

Esmé J. Baan ${ }^{1}$, Maria de Ridder ${ }^{1}$, Marjolein Engelkes ${ }^{1}$, Elisabeth Svensson ${ }^{2}$, Daniel PrietoAlhambra ${ }^{3}$, Francesco Lapi $^{4}$, Carlo Giaquinto ${ }^{5}$, Gino Picelli ${ }^{5}$, Frank Albers ${ }^{6}$, Lee Evitt ${ }^{7}$, Eric Bradford $^{8}$, Melissa K van Dyke ${ }^{9}$, Peter Rijnbeek ${ }^{1}$, Miriam Sturkenboom ${ }^{10}$, Hettie M Janssens ${ }^{11}$ and Katia M C Verhamme ${ }^{1}$

1 Department of Medical Informatics, Erasmus Medical Center, Rotterdam, Netherlands

2 Department of Clinical Epidemiology, Aarhus University hospital, Aarhus, Denmark

3 GREMPAL Research Group, Idiap Jordi Gol Primary Care Research Institute, Universitat Autonoma de Barcelona, Barcelona, Spain

4 Health Search, Italian College of General Practitioners and Primary Care, Florence, Italy

5 Pedianet, Padova, Italy

6 Global Respiratory Franchise, GlaxoSmithKline, Research Triangle Park, North Carolina, United

States of America

7 Research and Development, GlaxoSmithKline, GSK House, London, United Kingdom

8 Research and Development, GlaxoSmithKline, Research Triangle Park, North Carolina, United

States of America

9 Real World Evidence and Epidemiology, GSK, Upper Providence, PA, United States of America

10 Department of Epidemiology, Julius Center, Utrecht University, Utrecht, Netherlands

11 Department of Pediatric Pulmonology, Erasmus Medical Center/ Sophia Children's Hospital, Rotterdam, Netherlands

Background: There is sparse real life evidence on the risk factors and incidence of asthma exacerbations (AE) in children.

Aims: To assess incidence and risk factors of $A E$ in children with moderate to severe asthma in real life.

Methods: Patients aged 5-17 years with $\geq 1$ year of follow-up were identified in six European electronic health record databases from the Netherlands, Italy, UK, Denmark and Spain in 20082013. Asthma was defined as $>1$ asthma prescription within 3 months of an asthma diagnose code, severe asthma also required use of high dose inhaled corticosteroids combined with controller therapy. AE was defined as need of oral steroids, emergency department visit or hospitalization for asthma. Risk factors for AE were estimated by multivariate Poisson regression analyses.

Results: The cohort consisted of 226, 343 asthma patients (mean age in the databases 7.2-14.8 years), contributing 723, 674 patient years (PY). The proportion of severe asthma ranged between 1.6-15.5\%. AE rates ranged between 17-188/1, $000 \mathrm{PY}$, and were higher in severe asthma patients (41-326/1, $000 \mathrm{PY})$. In most databases girls had a higher AE rate than boys from adolescence on, and vice versa in young children. The risk was mainly increased by previous $A E$ (incidence rate: 3-29), and also by atopy and high blood eosinophilia ( $\geq 300$ cells/uL). Obesity was a risk factor of $A E$ in some databases.

Conclusions: In a real world setting, we showed high AE rates, with highest rates in children with severe asthma. Risk of exacerbation depended on age, gender, comorbidities and previous AE. Asthma management focusing on prevention of $A E$ is important to reduce the burden of asthma. Funding: GSK funded (PRJ2284) 